\title{
PO2-7-5
}

Poster session

\section{Study on the mechanism of Iridoid Glycosides in Radix Rehmanniae and Cornus Officinalis intervention in Diabetic Nephropathy}

\author{
Huiqin Xu, Xin Lv, Yuping Chen, Gaohong Lv, Yingxue Fu, Wenqing Bian \\ College of Pharmacy, Nanjing University of Chinese Medicine, China
}

Object:Diabetic Nephropathy (DN) is one of serious microvascular complications of diabetes.AGEs/RAGE signaling pathway plays an important role in mediating the pathological changes of inherent cellular structure and function in kidney,among which damage induced by AGEs of human renal mesangial cells (HRMCs) is extremely important.Radix rehmanniae (RR) and Cornus officinalis (CO) were used to treat diabetes mellitus and its complications for long in traditional Chinese medicine (TCM).In our study,AGEs-induced HRMC injury was used to study the effect and mechanism of RR and CO in DN focusing on AGEs-RAGE pathway.It would provide scientific basis for the clinical TCM in DN.Method:AGEs $(200 \mathrm{mg} / \mathrm{L})$ was used to induce HRMCs damage.Furthermore, different concentrations $(0.1,1,10 \mathrm{umol} / \mathrm{L})$ of iridoid glycosides from RR and $\mathrm{CO}$ were used to intervene the effect of AGEs in HRMCs. Phosphorylation of SphK1,P38MAPK,PTKs,MEK1/2,ERK1/2 and NF-/kB,protein levels of RAGE,Ras,S1P2,TGF,ROS, NOX4 and nuclear NF-kB were detected using western blot.Then the downstream signaling proteins of RAGE were suppressed by inhibitor (PF543,VX-11e,PDTC) on the basis of RAGE overexpression to investigate the molecular mechanism.The affinity of drugs and targets were investigated with biofilm interference technique.Results:Iridoid glycosides from Radix rehmanniae and Cornus officinalis could attenuate the AGEs-induced HRMCs damage significantly.Catalpol,acteoside from RR,loganin,morroniside from CO can reduce the phosphorylation of SphK1, P38MAPK,PTKs,MEK1/2,ERK1/2 and NF-kB.Protein expression of RAGE,S1P2,TGF,ROS,NOX4 and nuclear NF-kB were also decreased.COL-IV and FN secretion decreased after RAGE overexpressed.However, PF543,VX-11e,PDTC can eliminate these effect.Loganin,morroniside,catalpol also show strong affinity with Sphk1,ERK1/2 and NF-kB respectively. Conclusions:Iridoid glycosides in RR and CO suppress the AGEs induced HRMCs proliferation,ECM secretion, AGEs/RAGE pathway activation.SphK1,ERK1/2 and NF-kB were the potential targets of loganin,morroniside,catalpol respectively.Furthermore,combination of effective parts in $\mathrm{RR}$ and $\mathrm{CO}$ could represent its advantage in treating $\mathrm{DN}$ via synergistic effect in AGEs/RAGE pathway. These data expound the dominance and scientificity of the compatibly using of TCM in clinical. 